\title{
Postprandial VLDL-triacylglycerol secretion is not suppressed in obese type 2 diabetic men
}

\author{
E. Søndergaard • L. P. Sørensen • I. Rahbek • \\ L. C. Gormsen • J. S. Christiansen • S. Nielsen
}

Received: 3 April 2012 / Accepted: 6 June 2012 /Published online: 30 June 2012

(C) Springer-Verlag 2012

\begin{abstract}
Aims/hypothesis Type 2 diabetes is characterised by insulin resistance and increased post-absorptive secretion of VLDLtriacylglycerol (VLDL-TAG). Whether postprandial suppression of endogenous VLDL-TAG secretion is abnormala finding that would link hyperlipidaemia and type 2 diabetes-remains unclear.

Methods Eight type 2 diabetic men and eight healthy men were studied before and after a fat-free test meal ( $40 \%$ of resting energy expenditure). VLDL-TAG kinetics were assessed using a primed-constant infusion of ex vivo labelled $\left[1-{ }^{14} \mathrm{C}\right]$ triolein VLDL-TAG using non-steadystate calculations.

Results Type 2 diabetic men had a higher basal VLDL-TAG secretion rate and concentration than healthy men (mean $\pm \mathrm{SD}$ secretion rate $137 \pm 61$ vs $78 \pm 30 \mu \mathrm{mol} / \mathrm{min}$, respectively [ $p=0.03$ ]; median concentration 1.03 [range $0.58-1.75$ ] vs 0.33 [0.13-1.14] mmol/1, respectively $[p<0.01])$. Postprandially, the VLDL-TAG secretion rate decreased in healthy men $(p<0.01)$, but remained unchanged in diabetic men $(p=0.47)$. The VLDL-TAG concentration increased in diabetic men and decreased in healthy men postprandially $(p<0.05)$. The difference in VLDL-TAG secretion rate between the two groups approached significance $(p=0.06)$ and the relative change in VLDL-TAG secretion rate was significantly
\end{abstract}

E. Søndergaard $(\bowtie) \cdot$ L. P. Sørensen $\cdot$ I. Rahbek $\cdot$

J. S. Christiansen $\cdot$ S. Nielsen

Department of Endocrinology and Internal Medicine,

Aarhus University Hospital,

Nørrebrogade 44,

8000 Aarhus C, Denmark

e-mail: esbens@dadlnet.dk

L. C. Gormsen

Department of Clinical Physiology and Nuclear Medicine,

Aarhus University Hospital,

Aarhus, Denmark different $(p=0.01)$ between the two groups. Basal VLDLTAG clearance was significantly lower in diabetic men (diabetic men 133 [49-390] $\mathrm{ml} / \mathrm{min}$; healthy controls 215 [137-933] $\mathrm{ml} / \mathrm{min}[p<0.05])$. After meal ingestion, clearance decreased in healthy men $(p=0.03)$, but was unchanged in diabetic men $(p=0.58)$.

Conclusions/interpretation Obese type 2 diabetic men have impaired postprandial suppression of VLDL-TAG secretion compared with lean healthy men, contributing to their postprandial lipaemia and hypertriacylglycerolaemia.

Keywords Postprandial · Tracer studies · Triacylglycerol · VLDL

$\begin{array}{ll}\text { Abbreviations } \\ \text { Apo } & \text { Apolipoprotein } \\ \text { CT } & \text { Computed tomography } \\ \text { DEXA } & \text { Dual X-ray absorptiometry } \\ \text { REE } & \text { Resting energy expenditure } \\ \text { SA } & \text { Specific activity } \\ \text { sc } & \text { Subcutaneous } \\ \text { TAG } & \text { Triacylglycerol }\end{array}$

\section{Introduction}

Elevated plasma triacylglycerol (TAG) is an independent risk factor for cardiovascular disease, and recent studies indicate a direct causal relationship [1-3]. In addition, hypertriacylglycerolaemia is an important part of diabetic dyslipidaemia, and the macrovascular complications of diabetes are strongly related to alterations in lipoprotein metabolism $[4,5]$. As the prevalence of obesity and diabetes increases, elevated plasma TAG is likely to become a common finding; insight into TAG metabolism is therefore warranted. 
It is well established that chronic hyperinsulinaemia, as observed in, for example, insulin-resistant individuals, is associated with an increase in VLDL-TAG production [6-8]. This is linked to the insulin-resistant state, as chronic hyperinsulinaemia in insulinoma does not display the same abnormalities [9]. In contrast, acute hyperinsulinaemia leads to a rapid inhibition of hepatic VLDL-TAG secretion, as evidenced from data stemming from in vitro studies, animal models and human studies [6-8, 10]. Some previous studies have shown that diabetic patients fail to downregulate secretion of large VLDL $\left(\mathrm{VLDL}_{1}\right)$ in an experimental setting with high-dose insulin infusion [11, 12], whereas we reported a preserved suppressive effect of hyperinsulinaemia on VLDL-TAG secretion [7]. The impact of meal-induced hyperinsulinaemia, on the other hand, is less well understood. Knowledge regarding postprandial changes in VLDL kinetics is mostly indirect and derived from studies using the hyperinsulinaemic clamp to mimic the rapid increase in insulin levels observed after a meal [6, 10, 12-14]. This approach has the obvious advantage that steady-state kinetics can be applied to tracer studies, yielding reproducible estimates of both VLDL-TAG production and insulin sensitivity. However, the clamp technique is a highly experimental situation that differs from the physiological and more subtle postprandial changes in hormonal milieu. To our knowledge, no studies exist that have measured VLDL-TAG kinetics during and after a test meal.

We have developed a method to produce a VLDL-TAG tracer based on ex vivo labelling of the TAG moiety of VLDL particles and subsequent reinfusion [8, 15, 16]. In addition to calculation of the VLDL-TAG kinetic variables of interest, the method allows, by tracing VLDL-TAGassociated fatty acids, the study of their metabolic fate (i.e. to what extent they are oxidised or deposited in adipose tissue) [8]. Moreover, it is possible to study dynamic VLDL kinetics in addition to steady-state kinetics with a constant-infusion protocol. After steady state is achieved, interventions can be performed with subsequent calculations of VLDL kinetics with the equations of Steele [17], modified for non-steady-state conditions [18]. This approach has previously been applied in NEFA tracer studies and been demonstrated to produce satisfactory estimates of NEFA turnover [19]. The effects of meal ingestion on VLDLTAG kinetics are dynamic, which necessitates the use of nonsteady-state equations.

In these studies we used a VLDL-TAG tracer to measure basal and postprandial VLDL-TAG secretion and clearance to provide additional insight into the mechanisms responsible for the postprandial hyperlipidaemia typically found in individuals with type 2 diabetes. The aim was to test the hypothesis that obese type 2 diabetic men have impaired postprandial suppression of VLDL-TAG secretion compared with lean healthy men. In addition, we wanted to assess the relationship with NEFA availability.

\section{Methods}

Participants The study protocol was approved by the local ethics committee and written informed consent was obtained from all participants. Eight men with type 2 diabetes $\left(\mathrm{BMI}>28 \mathrm{~kg} / \mathrm{m}^{2}\right)$ with a diabetes duration of less than 5 years to ensure preserved insulin production, and eight healthy lean men (BMI $<25 \mathrm{~kg} / \mathrm{m}^{2}$ ) with no family history of type 2 diabetes, matched for age, were recruited from the outpatient clinic and through local advertisements. All were nonsmokers and at stable weight, and none regularly engaged in vigorous exercise. Normal blood count and thyroid, liver and kidney function were confirmed for all participants. Current diabetes treatments were lifestyle modifications alone in three patients and metformin, sulfonylurea or both in five patients. Oral hypoglycaemic agents were discontinued 3 weeks before the study day, while statin and antihypertensive therapies were discontinued 2 weeks before the study day. $\mathrm{HbA}_{1 \mathrm{c}}$ was measured prior to discontinuation of glucose-lowering medication.

Protocol One week before the metabolic study day, volunteers who met the eligibility criteria visited the clinical research centre after an overnight fast of 10-12 h. A blood sample was drawn for labelling of VLDL-TAG. A dual x-ray absorptiometry (DEXA) scan and abdominal computed tomography (CT) scan at the $\mathrm{L}_{2}-\mathrm{L}_{3}$ interspace were performed in order to obtain anthropometric indices [20]. Resting energy expenditure (REE) was measured by indirect calorimetry and individuals were interviewed by a dietitian. Based on REE and the dietitian's calculations, participants were provided with a weight-maintaining diet (55\% carbohydrate, $15 \%$ protein and $30 \%$ fat) by the hospital kitchen during the 3 days preceding the metabolic study.

The study protocol is illustrated in Fig. 1. Participants were admitted to the clinical research centre at 22:00 hours on the evening before the study. After an overnight fast, catheters were placed in an antecubital vein and a contralateral heated hand vein in order to obtain arterialised blood. Except for the test meal, only ingestion of tap water was allowed. The study day included a $2.5 \mathrm{~h}$ basal period $(-150$ $0 \mathrm{~min}$ ) and a $4.5 \mathrm{~h}$ postprandial period (0-270 $\mathrm{min})$. At $t=0$ to $15 \mathrm{~min}$ the participant consumed a fat-free test meal $(40 \%$

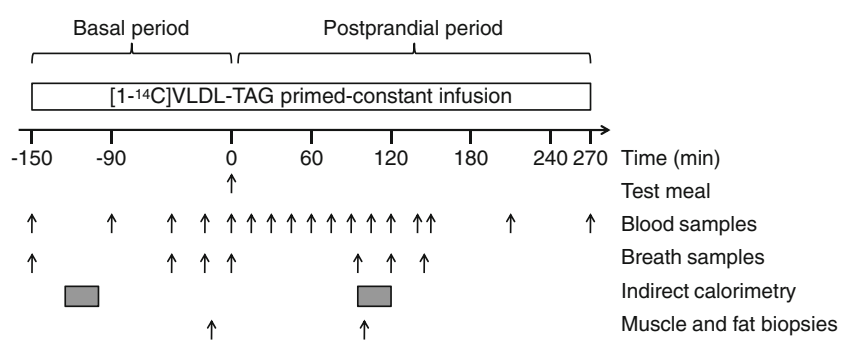

Fig. 1 Study protocol 
of REE [see below]). At $t=-150$ a primed-constant infusion of the individual's own $\left[1-{ }^{14} \mathrm{C}\right]$ triolein-labelled VLDL (priming with a bolus of $20 \%$ of labelled VLDL) was started. The antecubital catheter was used for VLDL-TAG tracer infusion and constant infusion of $\left[9,10-{ }^{3} \mathrm{H}\right]$ palmitate for $1 \mathrm{~h}$ in both the basal period ( $-90-30 \mathrm{~min}$ ) and during the postprandial period (60-120 $\mathrm{min}$ ). The other catheter was used for drawing blood samples to determine VLDL-TAG-specific activity (SA) (at $t=-150,-60,-30,-10,0,15,30,45,60,75,90,100,120$, 135, 150, 210 and $270 \mathrm{~min}$ ). Apolipoprotein (Apo)B-48 concentration was measured at $t=-30,90$ and $150 \mathrm{~min}$. After blood sampling at $270 \mathrm{~min}$, all catheters were removed and the participants were discharged.

VLDL-TAG tracer preparation A $60 \mathrm{ml}$ fasting blood sample was obtained under sterile conditions from each volunteer. Plasma was immediately separated and transferred to sterile Optiseal test tubes (Beckman Instruments, Brea, CA, USA), containing $740 \mathrm{KBq}$ of $\left[1-{ }^{14} \mathrm{C}\right]$ triolein (PerkinElmer, Waltham, MA, USA) dissolved in $200 \mu$ ethanol. Plasma containing labelled triolein was then sonicated in an incubator at $37^{\circ} \mathrm{C}$ for $6 \mathrm{~h}$. In order to isolate $\left[1-{ }^{14} \mathrm{C}\right]$ trioleinlabelled VLDL particles, labelled plasma was then transferred into Optiseal tubes (Beckman Instruments), covered with an $\mathrm{NaCl}$ solution $(d=1.006 \mathrm{~g} / \mathrm{ml})$, and centrifuged (50.3 rotor; Beckman Instruments) for $18 \mathrm{~h}$ at $98,350 \mathrm{rpm}$ and $10^{\circ} \mathrm{C}$. The supernatant fraction containing the labelled VLDL fraction was finally removed with a modified Pasteur pipette, and the solution was then passed through a Millipore filter (pore size diameter $=0.22 \mu \mathrm{m}$ [Millipore, Billerica, MA, USA]) and stored under sterile conditions at $5^{\circ} \mathrm{C}$. All samples were cultured to ensure sterility. Using this procedure, $\sim 10 \%$ of the tracer $(\sim 74 \mathrm{KBq})$ was recovered and available for infusion in each individual.

Plasma VLDL-TAG concentration and SA VLDL was isolated from plasma by ultracentrifugation. Approximately $3 \mathrm{ml}$ of each plasma sample was transferred into centrifuge tubes, covered with $\mathrm{NaCl}$ solution and centrifuged as described above. The top layer, containing VLDL, was obtained by slicing the tube $\sim 1 \mathrm{~cm}$ from the top using a tube slicer (Beckman Instruments), and the exact volume was recorded. A small proportion was analysed for TAG content, and the plasma concentration of VLDL-TAG was calculated. The remaining VLDL-TAG was transferred to a scintillation glass vial and $10 \mathrm{ml}$ of scintillation liquid was added. ${ }^{14} \mathrm{C}$ activity was measured using a liquid scintillation counter (Wallac 1409, PerkinElmer). A dual-channel program was used; counts were automatically corrected for quench and counted to a $<2 \%$ counting error. To determine the tracer infusion rate, $1 \mathrm{ml}$ of the tracer infusate was transferred to a scintillation glass vial, $10 \mathrm{ml}$ of scintillation liquid added and ${ }^{14} \mathrm{C}$ activity counted as described above.
Palmitate turnover Systemic palmitate flux was measured using an isotope dilution technique with a constant infusion of $\left[9,10-{ }^{3} \mathrm{H}\right]$ palmitate (Department of Nuclear Medicine, Aarhus University Hospital, Aarhus, Denmark) [21]. Plasma palmitate concentration and SA were measured at baseline and $10 \mathrm{~min}$ intervals over the last $30 \mathrm{~min}$ of the infusion periods by HPLC using $\left[{ }^{2} \mathrm{H}_{31}\right]$ palmitate as an internal standard. Steady-state SA was verified for each individual in the basal period, and palmitate flux $(\mu \mathrm{mol} / \mathrm{min})$ was calculated as $\left[9,10-{ }^{3} \mathrm{H}\right]$ palmitate infusion rate divided by the steadystate palmitate SA. In the postprandial period, palmitate flux was calculated using non-steady-state equations as previously described [19]

Body composition Total body fat, leg fat, fat percentage and fat-free mass were measured by DEXA (QDR-2000, Hologic, Bedford, MA, USA). Upper-body and visceral fat masses were assessed using CT measures of intra-abdominal and subcutaneous (sc) adipose tissue combined with abdominal fat mass measured by DEXA as previously described [20]. Upper-body sc fat was calculated as upper-body fat (DEXA) - visceral fat. Leg fat was measured using the region-of-interest program with the DEXA instrument.

Indirect calorimetry REE was measured by indirect calorimetry (Deltatrac monitor, Datex Instrumentarium, Helsinki, Finland). The initial $5 \mathrm{~min}$ was used for acclimatisation to the hood.

Test meal The test meal was an almost-fat-free breakfast designed by a dietitian ( $40 \%$ of REE; $85 \%$ carbohydrate, $10 \%$ protein and $5 \%$ fat) to avoid meal-fat interference with VLDL-TAG measurements. It consisted of fresh fruit, cornflakes, crispbread, jam and skimmed milk.

Laboratory procedures Plasma glucose was analysed using an YSI 2300 STAT Plus (YSI, Yellow Spring, OH, USA). TAG concentrations were analysed using a COBAS Fara II (F. Hoffmann-La Roche, Basal, Switzerland). Serum insulin concentrations were measured with an immunoassay (DAKO Denmark, Glostrup, Denmark). Serum NEFA concentrations were determined by a colorimetric method using a commercial kit (Wako Pure Chemical Industries, Osaka, Japan). ApoB-48 concentrations were analysed using an ELISA assay (Kamiya Biomedical Company, Seattle, WA, USA)

Calculations Calculations of VLDL-TAG kinetics were made using the equations of Steele [17], modified for nonsteady-state conditions [18]. A fractional volume of $1.4 \times$ plasma volume (5.5\% of body weight) was assumed. This is in accordance with our previous findings of the readily accessible pool size of VLDL-TAG [22]. VLDL-TAG concentrations and SA were spline fitted to minimise analytical variation that may 
occur when raw data are used in non-steady-state equations (Sigmaplot 11.0, Systat Software, Chicago, IL, USA) [23].

VLDL-TAG secretion and clearance were calculated according to the following equations $[17,18]$ :

VLDL - TAG secretion rate

$$
=\frac{F-\mathrm{p} V \times\left(\frac{\mathrm{c} t 1+\mathrm{c} t 2}{2}\right) \times\left(\frac{\mathrm{SA} t 2-\mathrm{SA} t 1}{t 2-t 1}\right)}{\frac{\frac{\mathrm{S} t 1+\mathrm{SA} t 2}{2}}{2}}
$$

VLDL - TAG clearance rate

$$
=\frac{\mathrm{VLDL}-\mathrm{TAG} \text { secretion rate }-\mathrm{p} V \times \frac{\mathrm{c} t 2-\mathrm{c} t 1}{t 2-t 1}}{\mathrm{c} 1}
$$

Where $F$ is the tracer infusion rate, $\mathrm{p} V$ is the fractional volume of distribution, $\mathrm{c} t$ is the concentration of tracee at time $t$, and SA $t$ is the specific activity at time $t$. The relative postprandial change in VLDL-TAG secretion was calculated in comparison to the pre-prandial steady-state secretion rate.

Statistics Data were analysed using SPSS 17.0 and expressed as mean $\pm \mathrm{SD}$ or median (range) as appropriate. Differences between groups were evaluated using Student's $t$ test or Mann-Whitney test. TAG and VLDL-TAG concentrations and VLDL clearance were log-transformed before analysis. Analyses of time-related effects between groups were performed using two-way ANOVA for repeated measurements with factors for group and time. Correlations were evaluated with Pearson's $r$ or Spearman's $\rho$ analyses. Statistical significance was set at $p=0.05$.

\section{Results}

Participant characteristics Participant characteristics are summarised in Table 1. The groups were well matched for age. As expected, diabetic men had a greater body weight, WHR and fat percentage. Also, a greater total, upper-body sc, lower-body sc and visceral fat mass were observed in the diabetic group. $\mathrm{HbA}_{1 \mathrm{c}}$ was somewhat greater in type 2 diabetic men, though not significantly so, demonstrating good metabolic control in the type 2 diabetic men $(6.2 \pm 0.7 \%$ vs $5.5 \pm 0.7 \%[p=0.10])$.

Insulin, glucose and NEFA concentrations As expected, type 2 diabetic men had higher fasting plasma glucose $(7.8 \pm 2.2 \mathrm{vs} 4.8 \pm 0.4 \mathrm{mmol} / 1[p<0.01])$ and higher fasting insulin $(65 \pm 24$ vs $21 \pm 10 \mathrm{pmol} / 1[p<0.001])$ than healthy men. After the test meal, the increases in glucose and insulin were greater and prolonged in diabetic men compared with healthy men (both $p<0.01$ ) (Fig. 2a,b). Basal NEFA levels were similar in diabetic and healthy men $(0.42 \pm 0.12$ vs $0.48 \pm 0.15 \mathrm{mmol} / 1[p=0.33])$ and in both groups NEFA were almost totally suppressed after $90 \mathrm{~min}$, with a slight delay in suppression in diabetic men $(p<0.01)$ (Fig. 2c).

Palmitate turnover Palmitate concentration $(130 \pm 20$ vs $135 \pm 32 \mu \mathrm{mol} / 1[p=0.68])$ and turnover $(174 \pm 26$ vs $215 \pm$ $61 \mu \mathrm{mol} / \mathrm{min}[p=0.11])$ were similar in the basal period in diabetic and healthy men, respectively. In the postprandial period, palmitate concentration $(41 \pm 22$ vs $23 \pm 7 \mu \mathrm{mol} / 1$ $[p<0.05])$ and turnover $(84 \pm 36 \mathrm{vs} 50 \pm 28 \mu \mathrm{mol} / \mathrm{min}[p=0.06])$ were suppressed significantly in both groups (both $p<0.01$ ). However, the degree of suppression was less pronounced in diabetic men compared with healthy men, both in absolute and relative terms (both $p=0.02$ ) (Fig. 2 d).

TAG and VLDL-TAG concentration As expected, greater basal total TAG and VLDL-TAG concentrations were observed in type 2 diabetic men compared with healthy men (TAG, $1.7[1.2-2.5]$ vs $0.9[0.7-1.6] \mathrm{mmol} / 1[p<0.01]$; VLDL-TAG $1.03[0.58-1.75]$ vs $0.33[0.13-1.14][p<0.01])$. After ingestion of the test meal total TAG concentration decreased in healthy men $(p<0.01)$, but remained unchanged in diabetic men $(p=0.17)$ (Fig. 3a). The effect of the test meal on total TAG was significantly different between the two groups $(p=0.01)$. Likewise, VLDL-TAG concentration over time was significantly different between the two groups after the test meal $(p<0.05)$, but did not change significantly in either diabetic men $(p=0.15)$ or healthy men $(p=0.12)$ (Fig. 3b).

VLDL-TAG secretion and clearance In the basal state, VLDL-TAG secretion rate was significantly greater in diabetic men compared with healthy men $(137 \pm 61$ vs $78 \pm$ $30 \mu \mathrm{mol} / \mathrm{min}[p=0.03])$. However, when corrected for fatfree mass, VLDL-TAG secretion was not significantly different between groups $(1.75 \pm 0.74$ vs $1.23 \pm 0.50 \mu \mathrm{mol} / \mathrm{min} / \mathrm{kg}$ $[p=0.12])$. Postprandially, VLDL-TAG secretion rate was suppressed by $52 \pm 21 \%(p<0.001)$ in healthy men, but remained unchanged in diabetic men $(p=0.47)$. Treatment vs time interaction tended to be different between groups, although not significantly $(p=0.06)$; i.e. the timerelated decrease in postprandial VLDL-TAG secretion was smaller in type 2 diabetic men (Fig. 4a). However, the relative suppression of VLDL-TAG secretion rate was significantly different between the two groups $(p=0.01)$, with impaired suppression in diabetic men (Fig. 4b). The basal VLDL-TAG clearance rate was significantly lower in diabetic men compared with healthy men (133 [49$390] \mathrm{ml} / \mathrm{min} ; 215$ [137-933] ml/min $[p<0.05])$. After meal ingestion, the VLDL-TAG clearance rate decreased in healthy men $(p=0.03)$, but was unchanged in diabetic men $(p=0.58)$, with no difference between groups $(p=0.21)$ (Fig. 4c). 
Table 1 Baseline characteristics of study participants
Data are mean $\pm \mathrm{SD}$ or median (range)

\begin{tabular}{llll}
\hline Characteristic & Type 2 diabetic men & Healthy men & $p$ value \\
\hline Participants (number) & 8 & 8 & 0.96 \\
Age (years) & $41(24-49)$ & $39(23-50)$ & $<0.01$ \\
Weight $(\mathrm{kg})$ & $110.5 \pm 13.3$ & $77.9 \pm 6.9$ & $<0.01$ \\
BMI $\left(\mathrm{kg} / \mathrm{m}^{2}\right)$ & $34.4 \pm 3.8$ & $22.9 \pm 1.4$ & $<0.01$ \\
WHR & $1.01 \pm 0.04$ & $0.89 \pm 0.04$ & $<0.01$ \\
Fat-free mass $(\mathrm{kg})$ & $77.7 \pm 5.8$ & $64.0 \pm 5.5$ & $<0.01$ \\
Total fat mass $(\mathrm{kg})$ & $32.9 \pm 9.2$ & $13.9 \pm 5.3$ & $<0.01$ \\
Fat percentage $(\%)$ & $29.3 \pm 5.1$ & $17.6 \pm 6.4$ & 0.01 \\
Visceral fat $(\mathrm{kg})$ & $4.2 \pm 1.0$ & $2.1 \pm 1.7$ & $<0.01$ \\
Upper-body sc fat $(\mathrm{kg})$ & $16.0 \pm 5.3$ & $5.1 \pm 3.3$ & $<0.01$ \\
Lower-body sc fat $(\mathrm{kg})$ & $9.8 \pm 2.6$ & $5.3 \pm 2.1$ & $<0.01$ \\
Triacylglycerol $(\mathrm{mmol} / \mathrm{l})$ & $2.1 \pm 0.6$ & $1.1 \pm 0.5$ & 0.07 \\
Total cholesterol $(\mathrm{mmol} / \mathrm{l})$ & $4.9 \pm 0.9$ & $4.1 \pm 0.7$ & 0.18 \\
LDL-cholesterol (mmol/l) & $2.9 \pm 1.0$ & $2.3 \pm 0.6$ & $<0.05$ \\
HDL-cholesterol (mmol/l) & $1.1 \pm 0.3$ & $1.3 \pm 0.3$ & 0.10 \\
HbA $(\%$ [mmol/mol]) & $6.2 \pm 0.7(44 \pm 8)$ & $5.5 \pm 0.7(37 \pm 8)$ & \\
\hline
\end{tabular}

ApoB-48 concentration For all participants and at all time points, ApoB-48 concentrations were below the assay detection limit of $7.8 \mathrm{pg} / \mathrm{ml}$.

Correlations No association between palmitate turnover and VLDL-TAG secretion rate was observed in either the basal or the postprandial period. The degree of suppression
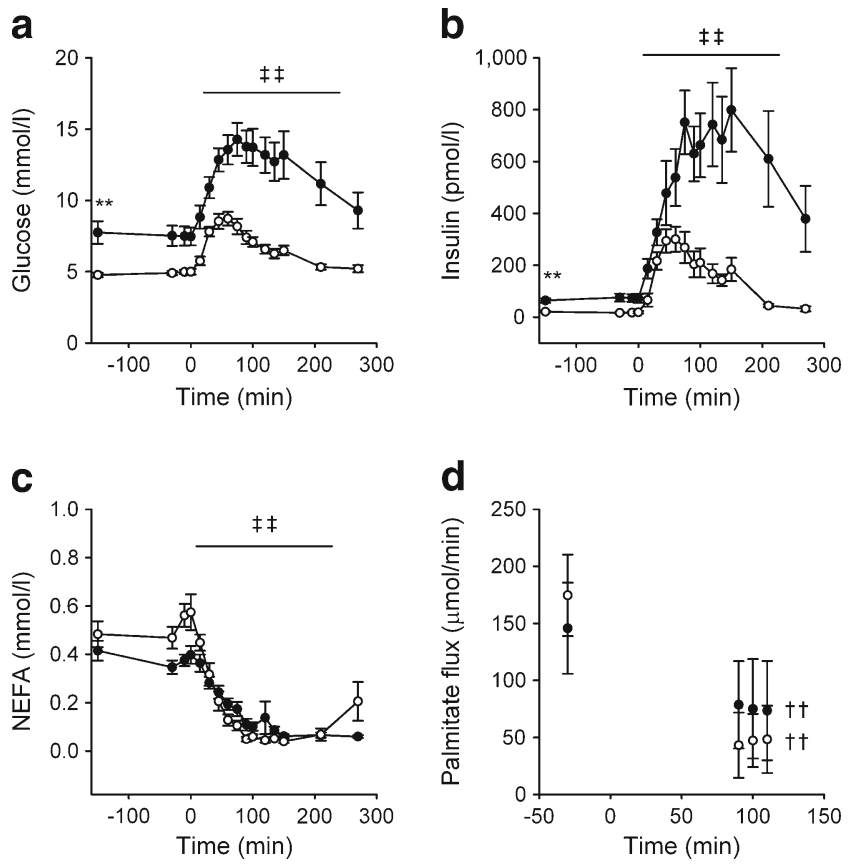

Fig. 2 Glucose (a), insulin (b), NEFA (c) and palmitate flux (d). Data are means \pm SEM. Black circles, type 2 diabetic men; white circles, healthy men. ${ }^{* *} p<0.01$ between groups in the basal period; ${ }^{\dagger \dagger} p<0.01$ for postprandial effect within group; ${ }^{*} p<0.01$ for postprandial effect (time $\times$ group) of palmitate turnover was significantly associated with the degree of suppression of VLDL-TAG secretion in diabetic men, both in absolute $(\rho=0.81, p=0.02)$ and relative $(\rho=0.74$, $p=0.04)$ terms. In healthy men, no association between suppression of palmitate turnover and VLDL-TAG secretion was observed. Visceral fat mass was not correlated with either basal VLDL-TAG secretion rate or absolute or relative suppression of VLDL-TAG in either of the two groups.

\section{Discussion}

To our knowledge, this is the first study to assess postprandial VLDL-TAG kinetics in type 2 diabetic men using a VLDL-TAG tracer. Our main finding was an abolition of postprandial suppression of VLDL-TAG secretion in type 2 diabetic men. Moreover, the VLDL-TAG clearance rate was significantly reduced postprandially in lean men, but remained
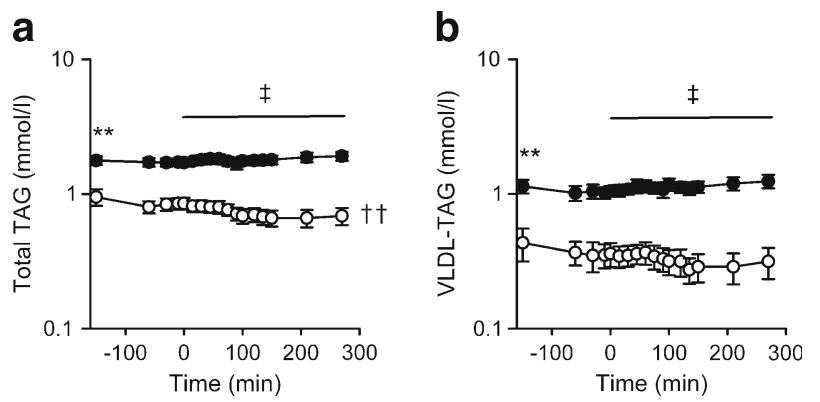

Fig. 3 Total TAG concentration (a) and VLDL-TAG concentration (b). Data are means \pm SEM. Black circles, type 2 diabetic men; white circles, healthy men. ${ }^{* *} p<0.01$ between groups in the basal period; ${ }^{\dagger} p<0.01$ for postprandial effect within group; ${ }^{\ddagger} p<0.05$ for postprandial effect (time $\times$ group) 


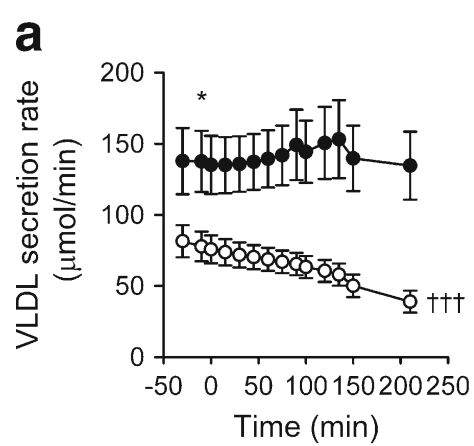

Fig. 4 VLDL-TAG secretion rate (a), relative change in VLDL-TAG secretion rate (b) and VLDL-TAG clearance (c). Data are means \pm SEM. Black circles, type 2 diabetic men; white circles, healthy men. ${ }^{*} p<0.05$

unchanged in type 2 diabetic men. However, a significant difference in the change in clearance could not be demonstrated between the two groups.

Type 2 diabetic patients are characterised by elevated postprandial concentrations of TAG, both in chylomicrons [24] and VLDL particles [25]. The increase in VLDL-TAG may be explained by increased VLDL-TAG secretion, decreased VLDL-TAG clearance or a combination of both. Postprandially, chylomicrons and VLDL mix in the blood and compete for the same clearance pathways. It has been shown that VLDL-TAG accumulates in human plasma after fat intake. The mechanism is explained by competition between chylomicrons and VLDL particles at the lipoprotein lipase (LPL) binding sites [26, 27]. However, studies have also shown an association between liver fat content and the postprandial increase in ApoB-100 [28, 29], indicating that endogenous VLDL-TAG secretion may also contribute to postprandial lipaemia in type 2 diabetes. Despite the wide range of different tracers and kinetic models employed to study VLDL-TAG kinetics, results in healthy individuals have convincingly shown that acute experimental hyperinsulinaemia decreases hepatic production of VLDL-ApoB [12, 14, 30-33] and VLDL-TAG [10, 12, 14, 30, 31], primarily through suppression of $\mathrm{VLDL}_{1}$ (rather than $\mathrm{VLDL}_{2}$ ) production $[12,32,33]$. Insulin-mediated suppression of VLDL-TAG secretion may partly be attributed to diminished hepatic NEFA delivery secondary to suppression of adipose tissue lipolysis [34]. However, as shown by Lewis et al, hyperinsulinaemia still suppresses VLDL-TAG secretion when NEFA levels are kept elevated by a concomitant infusion of heparin and intralipid [14]. This overriding suppressive effect of insulin on hepatic VLDL-TAG secretion appears physiologically beneficial by reducing competition between VLDL and chylomicron particles for peripheral TAG clearance and thereby minimising the transient postprandial hyperlipidaemia.

By using a unique VLDL-TAG tracer technique, the present study is the first to compare postprandial VLDLTAG secretion in healthy lean men and type 2 diabetic men. We report a blunted suppression of VLDL-TAG secretion

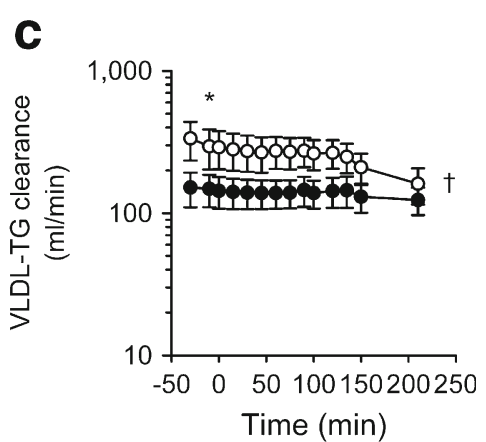

between groups in the basal period; ${ }^{\dagger} p<0.05$ for postprandial effect within group; ${ }^{\dagger \dagger} p<0.001$ for postprandial effect within group; ${ }^{\star} p<0.05$ for postprandial effect (time $\times$ group)

rate in type 2 diabetic men following a fat-reduced test meal, whereas a significant suppression was noted in healthy lean men. This was observed despite a greater increase in insulin in type 2 diabetic men compared with healthy men. We propose that this difference may contribute to postprandial hypertriglycerolaemia in type 2 diabetes.

One likely mechanism that may explain at least some of the observed difference is impaired postprandial insulinmediated suppression of VLDL-TAG secretion. Previous studies of the effect of hyperinsulinaemia on VLDL-TAG secretion in type 2 diabetes have been inconsistent with reports showing impaired [11,12] or preserved [7] suppression. This may be attributed to the prolonged and supraphysiological exposure to insulin during hyperinsulinaemic clamps, which may override the impaired suppression in type 2 diabetes at more physiological insulin concentrations. Moreover, meal ingestion is a more complex intervention, resulting in changes in several other substrate and hormone fluxes, which may also affect VLDL-TAG secretion. In all individuals, plasma ApoB- 48 concentrations were below the assay detection limit, which excludes chylomicron appearance as an explanation for the observed differences [35].

Diabetic men had a slower and less pronounced inhibition of lipolysis in response to the test meal. The difference in total NEFA concentration was minimal, but palmitate turnover was not suppressed to the same extent in diabetic men compared with healthy men. In addition, previous studies of hepatic NEFA delivery have demonstrated a greater contribution of visceral fat lipolysis with progressively greater visceral fat mass [36]. However, as both NEFA concentration and palmitate turnover were suppressed significantly in diabetic men this does not appear to be the most likely explanation for our findings. Furthermore, a significant association between suppression of palmitate turnover and VLDL-TAG secretion was noted in type 2 diabetic men, but not in healthy men. We cannot exclude the possibility that reduced availability of postprandial NEFA results in reduced VLDL-TAG secretion in our type 2 diabetic men. However, we find it more likely that the 
association in the diabetic men reflects the simultaneous failure to suppress both lipolysis and VLDL-TAG secretion.

We observed no postprandial change in VLDL-TAG clearance in diabetic men, whereas in healthy men a decrease was observed $2 \mathrm{~h}$ after meal ingestion. The latter finding may be due to a downregulation of VLDL-TAG clearance as a response to a decrease in plasma VLDLTAG concentration. Previously, no differences in wholebody VLDL-TAG clearance during a hyperinsulinaemic clamp have been observed in healthy or diabetic individuals [7, 11, 33, 37], perhaps because of a shift from VLDL-TAG fatty acid oxidation during fasting to storage during hyperinsulinaemia resulting in no net change in the total clearance rate. The higher basal clearance in healthy men compared with diabetic men in the present study is in line with previous findings from our group showing a tendency towards lower clearance in type 2 diabetic men vs weight-matched controls [7].

During recent years it has been argued that non-fasting TAG levels should replace fasting levels in assessing cardiovascular disease [38]. This proposed change in recommendations is based on reports indicating a higher predictive value of non-fasting vs fasting TAG $[39,40]$. When non-fasting levels of TAG are measured, postprandial VLDL-TAG regulation plays an important role as VLDL particles compete with chylomicrons for lipolysis of their content of lipids [26, 27]. Non-fasting TAG levels therefore also reflect the suppressive effect of insulin on hepatic VLDL-TAG secretion and may be a valid indicator of insulin resistance.

There are limitations to our study. First, the effect of meal ingestion is a dynamic response rendering it impossible to study in a steady state. Therefore, we used non-steady-state equations to calculate kinetic variables. This requires assumptions regarding the readily accessible pool size. We used results from a previous study from our group in which pool size was determined in healthy individuals [22]; it may not necessarily be the same for diabetic men. Second, the VLDL-TAG tracer was prepared from plasma obtained in the postabsorptive state and, as VLDL particle composition changes in response to hyperinsulinaemia, the VLDL-TAG tracer used to determine VLDL-TAG kinetics during hyperinsulinaemia should ideally have been prepared from plasma obtained during hyperinsulinaemia. However, Lewis et al found no differences in VLDL-ApoB kinetics in plasma drawn under basal and hyperinsulinaemic conditions [31]. Furthermore, our tracer-labelling procedure could alter the composition of the VLDL particles, thereby affecting our kinetic estimates. However, we have previously shown a similar metabolic capacity of our tracer to native VLDLTAG [16]. Third, as our study groups differed not only in the presence and absence of diabetes but also in body weight and body composition, as well as biochemical variables (e.g. insulin, lipids and glucose), we cannot conclude that the observed differences in VLDL-TAG kinetics are caused by the type 2 diabetic state and/or insulin resistance per se. In order to assess the impact of these additional differences, studies including a weight-matched control group are required. Finally, we cannot automatically extend our findings to women.

In conclusion, obese type 2 diabetic men have impaired postprandial suppression of VLDL-TAG secretion compared with lean healthy men, which probably contributes to their postprandial lipaemia and hypertriacylglycerolaemia.

Acknowledgements The authors acknowledge the excellent technical assistance of L. Kvist and S. Sørensen of the Medical Research Laboratories, Aarhus University Hospital, Denmark

Funding This work was supported by grants from the Danish Medical Research Council, the Novo Nordisk Foundation and the Danish Diabetes Foundation (to S. Nielsen).

Duality of interest The authors declare that there is no duality of interest associated with this manuscript.

Contribution statement ES designed the study, acquired, analysed and interpreted data and drafted the article. LPS and IR acquired and interpreted data and revised the article. LCG and SN conceived and designed the study, interpreted data and revised the article. JSC interpreted data and revised the article. All authors approved the final version.

\section{References}

1. Hokanson JE, Austin MA (1996) Plasma triglyceride level is a risk factor for cardiovascular disease independent of high-density lipoprotein cholesterol level: a meta-analysis of population-based prospective studies. J Cardiovasc Risk 3:213-219

2. Austin MA, Hokanson JE, Edwards KL (1998) Hypertriglyceridemia as a cardiovascular risk factor. Am J Cardiol 81:7B-12B

3. Triglyceride Coronary Disease Genetics Consortium and Emerging Risk Factors Collaboration (2010) Triglyceride-mediated pathways and coronary disease: collaborative analysis of 101 studies. Lancet 375:1634-1639

4. Adler AI, Stevens RJ, Neil A, Stratton IM, Boulton AJ, Holman RR (2002) UKPDS 59: hyperglycemia and other potentially modifiable risk factors for peripheral vascular disease in type 2 diabetes. Diabetes Care 25:894-899

5. Koskinen P, Manttari M, Manninen V, Huttunen JK, Heinonen OP, Frick MH (1992) Coronary heart disease incidence in NIDDM patients in the Helsinki Heart Study. Diabetes Care 15:820-825

6. Lewis GF, Steiner G (1996) Acute effects of insulin in the control of VLDL production in humans. Implications for the insulinresistant state. Diabetes Care 19:390-393

7. Sørensen LP, Andersen IR, Søndergaard E et al (2010) Basal and insulin mediated VLDL-triglyceride kinetics in type 2 diabetic men. Diabetes 60:88-96

8. Gormsen LC, Nellemann B, Sorensen LP, Jensen MD, Christiansen JS, Nielsen S (2009) Impact of body composition on very-lowdensity lipoprotein-triglycerides kinetics. Am J Physiol Endocrinol Metab 296:E165-E173 
9. Duvillard L, Florentin E, Fdr P et al (2011) Endogenous chronic hyperinsulinemia does not increase the production rate of VLDL apolipoprotein B: proof from a kinetic study in patients with insulinoma. J Clin Endocrinol Metabol 96:2163-2170

10. Shumak SL, Zinman B, Zuniga-Guarjardo S, Poapst M, Steiner G (1988) Triglyceride-rich lipoprotein metabolism during acute hyperinsulinemia in hypertriglyceridemic humans. Metabolism $37: 461-466$

11. Malmstrom R, Packard CJ, Caslake M et al (1997) Defective regulation of triglyceride metabolism by insulin in the liver in NIDDM. Diabetologia 40:454-462

12. Adiels M, Westerbacka J, Soro-Paavonen A et al (2007) Acute suppression of VLDL1 secretion rate by insulin is associated with hepatic fat content and insulin resistance. Diabetologia 50:23562365

13. Mittendorfer B, Patterson BW, Klein S, Sidossis LS (2003) VLDLtriglyceride kinetics during hyperglycemia-hyperinsulinemia: effects of sex and obesity. Am J Physiol Endocrinol Metab 284: E708-E715

14. Lewis GF, Uffelman KD, Szeto LW, Weller B, Steiner G (1995) Interaction between free fatty acids and insulin in the acute control of very low density lipoprotein production in humans. J Clin Invest 95:158-166

15. Gormsen LC, Jensen MD, Schmitz O, Moller N, Christiansen JS, Nielsen S (2006) Energy expenditure, insulin, and VLDLtriglyceride production in humans. J Lipid Res 47:2325-2332

16. Gormsen LC, Jensen MD, Nielsen S (2006) Measuring VLDLtriglyceride turnover in humans using ex vivo-prepared VLDL tracer. J Lipid Res 47:99-106

17. Steele R (1959) Influences of glucose loading and of injected insulin on hepatic glucose output. Ann NY Acad Sci 82:420-430

18. De Bodo RC, Altszuler N, Dunn A, Steele R, Armstrong DT, Bishop JS (1959) Effects of exogenous and endogenous insulin on glucose utilization and production. Ann NY Acad Sci 82:431-451

19. Jensen MD, Heiling V, Miles JM (1990) Measurement of nonsteady-state free fatty acid turnover. Am J Physiol Endocrinol Metab 258:E103-E108

20. Jensen MD, Kanaley JA, Reed JE, Sheedy PF (1995) Measurement of abdominal and visceral fat with computed tomography and dual-energy x-ray absorptiometry. Am J Clin Nutr 61:274-278

21. Guo Z, Nielsen S, Burguera B, Jensen MD (1997) Free fatty acid turnover measured using ultralow doses of [U-13C]palmitate. J Lipid Res 38:1888-1895

22. Sorensen LP, Gormsen LC, Nielsen S (2009) VLDL-TG kinetics: a dual isotope study for quantifying VLDL-TG pool size, production rates, and fractional oxidation in humans. Am J Physiol Endocrinol Metab 297:E1324-E1330

23. Wolfe RR, Peters EJ, Klein S, Holland OB, Rosenblatt J, Gary H (1987) Effect of short-term fasting on lipolytic responsiveness in normal and obese human subjects. Am J Physiol Endocrinol Metab 252:E189-E196

24. Annuzzi G, de Natale C, Iovine $C$ et al (2004) Insulin resistance is independently associated with postprandial alterations of triglyceriderich lipoproteins in type 2 diabetes mellitus. Arterioscler Thromb Vasc Biol 24:2397-2402
25. Hodson L, Bickerton AST, McQuaid SE et al (2007) The contribution of splanchnic fat to VLDL triglyceride is greater in insulinresistant than insulin-sensitive men and Women. Diabetes $56: 2433-2441$

26. Schneeman BO, Kotite L, Todd KM, Havel RJ (1993) Relationships between the responses of triglyceride-rich lipoproteins in blood plasma containing apolipoproteins B-48 and B-100 to a fat-containing meal in normolipidemic humans. Proc Natl Acad Sci USA 90:2069-2073

27. Cohn JS, Johnson EJ, Millar JS et al (1993) Contribution of apoB48 and apoB-100 triglyceride-rich lipoproteins (TRL) to postprandial increases in the plasma concentration of TRL triglycerides and retinyl esters. J Lipid Res 34:2033-2040

28. Tushuizen ME, Pouwels PJ, Bontemps S et al (2010) Postprandial lipid and apolipoprotein responses following three consecutive meals associate with liver fat content in type 2 diabetes and the metabolic syndrome. Atherosclerosis 211:308-314

29. Matikainen N, Manttari S, Westerbacka J et al (2007) Postprandial lipemia associates with liver fat content. J Clin Endocrinol Metab 92:3052-3059

30. Lewis GF, Zinman B, Uffelman KD, Szeto L, Weller B, Steiner G (1994) VLDL production is decreased to a similar extent by acute portal vs peripheral venous insulin. Am J Physiol Endocrinol Metab 267:E566-E572

31. Lewis GF, Uffelman KD, Szeto LW, Steiner G (1993) Effects of acute hyperinsulinemia on VLDL triglyceride and VLDL apoB production in normal weight and obese individuals. Diabetes 42:833-842

32. Malmstrom R, Packard CJ, Caslake M et al (1998) Effects of insulin and acipimox on VLDL1 and VLDL2 apolipoprotein B production in normal subjects. Diabetes 47:779-787

33. Malmstrom R, Packard CJ, Watson TD et al (1997) Metabolic basis of hypotriglyceridemic effects of insulin in normal men. Arterioscler Thromb Vasc Biol 17:1454-1464

34. Lewis GF (1997) Fatty acid regulation of very low density lipoprotein production. Curr Opin Lipidol 8(3):146-153

35. Robertson MD, Parkes M, Warren BF et al (2003) Mobilisation of enterocyte fat stores by oral glucose in humans. Gut 52:834-839

36. Nielsen S, Guo Z, Johnson CM, Hensrud DD, Jensen MD (2004) Splanchnic lipolysis in human obesity. J Clin Invest 113:15821588

37. Cummings MH, Watts GF, Umpleby AM et al (1995) Acute hyperinsulinemia decreases the hepatic secretion of very-lowdensity lipoprotein apolipoprotein B-100 in NIDDM. Diabetes 44:1059-1065

38. Nordestgaard BG, Langsted A, Freiberg JJ (2009) Nonfasting hyperlipidemia and cardiovascular disease. Curr Drug Targets 10:328-335

39. Nordestgaard BG, Benn M, Schnohr P, Tybjaerg-Hansen A (2007) Nonfasting triglycerides and risk of myocardial infarction, ischemic heart disease, and death in men and women. JAMA 298:299308

40. Bansal S, Buring JE, Rifai N, Mora S, Sacks FM, Ridker PM (2007) Fasting compared with nonfasting triglycerides and risk of cardiovascular events in women. JAMA 298:309-316 